

\section{ÍNDICE}

\section{ÁMBITOS PERSONALES PERSONALÁMBITOS}

Crisis de Venezuela: Análisis y perspectivas según los titulares de la prensa argentina, española y china

Venezuela's crisis: Analysis and perspectives in the headlines of Argentine, Spain and China press

Hui Feng Liu

Tratamiento informativo de la violencia de género: asesinatos de mujeres. Análisis de la agencia EFE

Informative treatment of gender violence: murders of women. Analysis of the press agency EFE Rosa Rodríguez Cárcela, Agustín López Vivas

MONOGRAFICOS MONOGRAPHS

Presentación Monográfico. El universo transmedia de los medios de comunicación universitarios: acción dentro y fuera del aula en la sociedad postdigital

Antonia Isabel Nogales-Bocio, Ángels Álvarez villa

El papel transformador de la radio universitaria en materias teóricas ajenas a la comunicación

The transformative role of college radio in theoretical subjects outside communication

Miguel Ángel Díaz Monsalvo

La radiodifusión universitaria: acción discursiva radiofónica para la divulgación de la ciencia University Radio Broadcasting: Radiophonic Discursive Action for the Science Popularization Jorge Sadi Durón, Joel Zapata Salazar

El uso corporativo de Instagram en las universidades privadas españolas. Estudio comparativo de treinta y cinco universidades

The corporate use of Instagram in spanish private universities. Comparative analysis of thirty-five private universities 
La radio universitaria como herramienta de inclusión social: OndaCampus en contextos como la cárcel y barrios desfavorecidos

The university radio as a tool for social inclusion: OndaCampus in contexts like the prison and disadvantaged neighborhoods

Leonor Real Adame, Daniel Martín-Pena, Macarena Parejo Cuéllar

Hacer radio universitaria en la era de YouTube: uso de la plataforma de vídeos a demanda en el contexto mexicano

Make college radio in the age YouTube: use of video on demand platform in the Mexican context Marina Vázquez Guerrero

\section{ARTÍCULOS ARTICLES}

O impresso e o digital nos modelos de negócios de jornais locais: uma análise do Sermos Galiza

The press and digital in business models from local media: Analysis of newspaper Sermos Galiza

Giovanni Ramos

Publicidad y cáncer en la prensa escrita (1903-1912)

Advertising and cancer in the written press (1903-1912)

Laura Almudéver-Campo, Ramón Camaño-Puig

Game rules vs. fandom. How Nintendo's Animal Crossing fan-made content negotiates the videogame meanings

Las reglas del juego vs. el fandom. Cómo el contenido hecho por fans de Nintendo Animal Crossing negocia los significados del videojuego

Jose A. Moreno

La comedia de situación y su análisis textual: evolución de los elementos constructivos del formato

The sitcom and its textual analysis: evolution of the constructive elements of the format

Darío Martín Sánchez

Análisis del discurso emocional de Donald Trump en la campaña electoral de $\mathbf{2 0 1 6}$ Analysis of Donald Trump's emotional speech on the 2016 election campaign 
Innovar, comunicar y transformar (en) la Universidad

Innovate, communicate and transform (at) the University

María Sofía Bernat

288-292

Transparencia en los medios: Un requisito imprescindible para medir la rentabilidad social en radio y televisión

Transparency in the media: An essential requirement to measuresocial profitability in radio and television

Amanda Salazar

Los estudios feministas en comunicación: representación de las mujeres en la revolución tecnológica

Feminist studies in communication: representation of women in the technological revolution 


\title{
El papel transformador de la radio universitaria en materias teóricas ajenas a la comunicación
}

\author{
The transformative role of college radio in theoretical subjects \\ outside communication
}

\author{
Miguel Ángel Díaz Monsalvo, Universidad Europea Miguel de Cervantes, \\ Calle del Padre Julio Chevalier, 2, 47012, Valladolid. \\ madiaz@uemc.es | Orcid: https://orcid.org/0000-0003-2896-551X
}

\author{
DOI: http://dx.doi.org/10.12795/Ambitos.2020.i47.04
}

\begin{abstract}
Resumen
Este estudio muestra la experiencia desarrollada con 99 estudiantes de la Universidad Europea Miguel de Cervantes de Valladolid. Los participantes divulgaron los resultados de investigaciones grupales desarrolladas en asignaturas de carácter teórico (Recursos Humanos, Teoría de la Comunicación e Historia) a través de podcasts, que se colgaron en la radio universitaria del centro. Tras las explicaciones de los conceptos teóricos, los docentes plantearon a los estudiantes un problema a investigar grupalmente $\mathrm{y}$, posteriormente, divulgar a través de la radio. Mediante una metodología mixta basada en el análisis de los podcasts, encuestas y entrevistas en profundidad a los implicados, tanto a estudiantes como docentes, se ha cotejado su grado de desarrollo de capacidades vinculadas a la comunicación, la viabilidad del proyecto y la configuración de esta herramienta tecnológica como aliada para afianzar el estudio de estas asignaturas. La mitad de los estudiantes carecían de experiencia radiofónica previa. Tras su ejecución, los alumnos aseguraron estar más implicados en la asignatura, de eminente carga teórica. También constataron que se habían esforzado por ser más divulgativos a pesar de carecer de apoyo visual durante las presentaciones. Las calificaciones de los tres docentes implicados corroboraron esa
\end{abstract}


percepción. La aportación trasluce que las radios universitarias ya no solo son un laboratorio de experimentación y adquisición de competencias para los estudiantes del área de Comunicación, sino que también son viables para expandir sus posibilidades y erigirse en herramientas de innovación docente, dirigiéndose a alumnos de otros Grados para mejorar sus habilidades comunicativas, claves para su desarrollo profesional.

\section{Abstract}

This study shows the experience carried out with 99 students of the European University Miguel de Cervantes, Valladolid. The participants shared with their partners the results of groupal researchs developed in theoretical subjects (Human Resources, Communication Theory and History) through podcasts, which were uploaded and shared on the college radio. After the professors explanations, the students must investigate about a real problem linked to the subject in order to be explained through the radio. Through a mixed methodology based on the analysis of podcasts, surveys and in-depth interviews with people involved, it has been analysed their communicational degree of development, the viability of the project and the college radio's potential to stand up as an ally to strengthen the study of those subjects. Half of the students lacked previous radio experience. After its execution, the students said they were more involved and interested in those theorical subjects. They also found that they had endeavored to be more informative despite lacking visual support. The qualifications of the three professors involved also corroborated that perception. The contribution shows that college radios are not only a laboratory for experimentation, testing, and acquisition of skills for students in the area of Communication, but are also viable to expand their possibilities and become a teaching innovation tool, also addressing students from other degrees to improve their communication skills, keys to their professional development.

Palabras clave: Radio universitaria, educación, podcast, innovación

Keywords: College radio, education, podcast, innovation

\section{INTRODUCCIÓN}

Las radios universitarias se destinan a estudiantes, sean o no de carreras vinculadas a la comunicación, apasionados por este medio y con ganas de transmitir contenidos que no suelen tener reflejo en las parrillas de las emisoras generalistas. Se trata de un fenómeno reciente con alto impacto en las universidades españolas, y el corpus teórico desarrollado en estos años ha configurado a este proyecto educativo como un 
laboratorio de ensayo y experimentación que refuerza las competencias para los estudiantes de cara a su futuro profesional.

En esta investigación se conjugan elementos tradicionales del proceso de enseñanzaaprendizaje (asignaturas con eminente carga teórica, evaluación por competencias, y ejecución de trabajos grupales), con otras actuales (radios universitarias o evaluación por rúbricas) para generar una perspectiva diferente. Los docentes de las asignaturas Recursos Humanos, Teoría de la Comunicación e Historia Política y Social, que se imparten en cuatro Grados (Periodismo, Comunicación Audiovisual, Publicidad y Administración y Dirección de Empresas) explicaron en el aula los conceptos teóricos de una parte del temario; posteriormente, solicitaron al alumnado realizar un trabajo grupal en el que debían investigar un problema actual en base a esos conceptos y trasladar los resultados de sus conclusiones en la radio universitaria. Los podcasts fueron grabados y evaluados, siendo la vertiente propiamente comunicativa uno de los ítems calificados.

El objetivo general de la investigación se cimienta en clarificar qué grado de desarrollo de habilidades vinculadas a la comunicación experimentan los estudiantes mediante la modificación de su hábitat académico habitual, al pasar del aula a la emisora.

Se plantea como hipótesis principal que los alumnos incrementan su motivación en asignaturas de carácter teórico al plantearles el reto de salir de su área de confort al tener que exponer sus resultados de investigación grupales en el medio radiofónico. Emana una hipótesis complementaria que gira en torno a que el alumnado incrementa su nivel de responsabilidad y exigencia al saber que su trabajo será divulgado en un medio de comunicación, simulando un ambiente real de trabajo y generando así una nueva dinámica de aprendizaje autónomo que afecta tanto al ámbito individual (exigencia de ser conciso) como grupal (coordinación entre integrantes para conformar un producto profesional que será emitido).

Se han empleado técnicas de investigación mixtas (encuestas a los alumnos y docentes implicados, análisis de las calificaciones obtenidas, y entrevistas a los profesores), que han permitido extraer, por un lado, datos cuantitativos (mediante media y moda estadística, correlacionando esos valores con las variables de sexo, edad, Grado a estudiar y experiencia previa en el medio radiofónico) y, por el otro, datos cualitativos acerca de la pertinencia, desarrollo, grado de utilidad y mejoras futuras de esta experiencia, sustentada en origen -aunque implementado novedadesen otras iniciativas realizadas en radios universitarias españolas (López Vidales y González, 2014). 


\section{LAS RADIOS UNIVERSITARIAS EN EL MODELO ESPAÑOL}

Aunque en España ha sido un fenómeno reciente, especialmente en lo relativo a su expansión, las radios universitarias tienen un siglo de vigencia y su nacimiento se sitúa a principios del siglo XX en Estados Unidos a través de las college radios (Ortiz Sobrino y Martín Pena, 2014). En el caso español, aunque las primeras experiencias datan de la década de 1970 en la UNED, no es hasta principios del siglo XX, al calor del auge de las Tecnologías de Información y Comunicación, cuando las universidades españolas entran en contacto con esta realidad.

Desde entonces, los estudios científicos han enmarcado a las estaciones radiofónicas universitarias como un proyecto educativo, sin ánimo de lucro, que se erige como laboratorio de experimentación y ensayo para los alumnos -no solo de los Grados vinculados a la comunicación- y un espacio donde poder contar aquellos contenidos que son de su interés y no tienen cabida en las radios convencionales, a través, en ocasiones, de fórmulas de desarrollo novedosas (Vázquez Guerrero, 2012; López Vidales, Gómez Rubio y Hernando Lera, 2015; Zamarra López, 2018). Y en el caso de los estudiantes de Periodismo y Comunicación Audiovisual, este campo de entrenamiento les sirve para desarrollar y consolidar aquellas competencias aprendidas en las aulas y que necesitarán en su incorporación al mercado laboral (Ortiz Sobrino, Marta Lazo, y Martín-Pena, 2016, Pinto y Vivas Moreno, 2016; y Díaz, 2018).

La esencia de la radio se construye en su capacidad de comunicación. Y en el ámbito educativo, la aplicación de la radio permite multiplicar las posibilidades. Desde hace medio siglo se han sentado las bases de la llamada educomunicación; un término, empero, que ya comenzó a dejar huella a mediados del siglo XIX a través de los esfuerzos investigadores del pedagogo Célestin Freinet, aunque no fue hasta un siglo después, a mediados del XX, cuando Mario Kaplún comenzó a explicar este fenómeno.

La Unesco, a través de varios encuentros celebrados en la década de los 70 , perfiló los pilares nominativos acerca de la educación en materia de comunicación: "todas las formas de estudiar, aprender y enseñar a todos los niveles (...) y en toda circunstancia, la historia, la creación, la utilización y la evaluación de los medios de comunicación como artes prácticas y técnicas" (Morsy, 1984, p.8). A este respecto, señala Pérez Tornero (2008) que "la comunicación y la educación no pueden seguir existiendo, del modo tradicional, como ámbitos totalmente separados. La sociedad de la información ha puesto en evidencia la necesidad de conectar ambos mundos, el educativo y el comunicativo" (p.2). Aun así, Barranquero (2007) matizó que dicha necesidad no se había trasladado al ámbito real por desconocimiento. 
Una radio universitaria tiene, como función adicional la "divulgación científica del conocimiento generado en su seno o de formación de sus alumnos" (Ponce López, 2019, p.193). Pinto (2016) amplía el espectro al considerar que es un lugar de convergencia para todo tipo de alumnos, no sólo los vinculados a estudios de Comunicación, en tanto que Sánchez Carrero y Contreras Pulido inciden en que "abarque a todos los sectores, principalmente a los más jóvenes, para concebir una figura del prosumidor verdaderamente empoderada ante una sociedad digital en continuo cambio" (2012, p.63). Por prosumidor (término profusamente explicado en investigaciones como las de López Vidales y Gómez Rubio, 2014) se entiende a aquella persona que participa activamente en el proceso de creación de contenidos radiofónicos, trascendiendo el mero rol de oyente. Se trata de un término que deviene de la fusión de productor y consumidor.

Señala López Conde (2019) que "la radio universitaria puede ser utilizada como instrumento de educación formal, con la realización de programas con fines docentes, complementarios a los planes de estudios establecidos" (p.194). Una emisora universitaria puede cumplir con este objetivo imbricándose con las directrices marcadas con el Espacio Europeo de Educación Superior, que pide nuevas fórmulas didácticas y esfuerzos docentes para mejorar el proceso de enseñanza-aprendizaje: "la importancia del aprendizaje colaborativo con respecto a la investigación enfocado a situaciones reales en las que los estudiantes presentan un interés particular de su área profesional" (Romero, Forero y Rodríguez, 2018, p.5). En este contexto, una emisora radiofónica de una universidad "permite salir de esquemas educativos lineales que ahora exigen las nuevas reformas educativas europeas" (Vázquez Guerrero, 2011, p.58).

Constatado el potencial educativo de la radio online al facilitar la escucha tanto en streaming como en podcast (Blanco, 2007; Gutiérrez y Rodríguez, 2010; Ortiz 2011; Perona 2011; López, Gómez y Hernando, 2016), las radios universitarias son atractivas para la docencia. Casos como Radio Unizar, Radio UNED, Uniradio, Onda Campus, Polimedia, y Radio Aula en Radio UVa han generado microespacios en radios generalistas, contenidos didácticos de apoyo a la docencia, o programas sobre temas complejos de actualidad poco accesibles y comprensibles por el gran público, pero adaptándolos a un estilo divulgativo.

\subsection{El potencial educativo del podcast}

Parejo Cuéllar, Vivas Moreno y Martín Pena (2018) han constatado escasa presencia de las Ciencias Sociales en los contenidos radiofónicos, algo que achacan a la dificultad de divulgación que tienen este tipo de contenidos frente a las disciplinas 
científicas, consideradas como más cercanas y operativas frente a la reflexión de las áreas humanísticas.

Para salvar el obstáculo de la rigurosa acotación de la radio destinada solo a estudiantes de Periodismo y Comunicación Audiovisual, Ortiz Sobrino (2008 y 2012) recuerda que el Espacio Europeo de Educación Superior postula la necesidad de que la preparación académica sea global y transversal, de tal modo que todo estudiante sepa asumir competencias que le permitan desarrollar trabajos prácticos en un entorno cambiante y tendente a lo transmedia. También, Chenovart y Castelló (2018) señalan que las emisoras universitarias permiten "formar y a unir a unos estamentos que en muchas ocasiones no tienen posibilidades de coincidir" (p.115).

Otros estudios, como los de Vargas-Guerrero junto a más de diez colaboradores (2018), han demostrado que la implantación del medio radiofónico como instrumento en proyectos pedagógicos "permiten desarrollar la capacidad de los estudiantes para sistematizar la información y dar coherencia desde el punto de vista radiofónico, al tiempo que incentivan la creatividad pertinente a los procesos inherentes al entorno educativo" (p.651).

Ruipérez, Gutiérrez Palmero y Olarte (2011) constataron "las ventajas, apenas explotadas, de la radio como instrumento de aprendizaje al convertir a los estudiantes de meros receptores en actores principales, reforzando indirectamente el perfil cultural y universitario" (p.846), y se añade un nuevo ingrediente, Internet, que ha permitido "una fórmula mixta de texto y de audiovisual que permite la captación de información y su utilización en el momento que desea el usuario". (De Semir, 2003, p.9)

Un binomio, radio e Internet, que debe ser aprovechado en el espectro de la educación superior, entroncándolo en el concepto de alfabetización mediática, entendida como

Conocimientos, actitudes y habilidades que un ciudadano, en este caso un docente universitario, debe dominar para ser competente en todas las dimensiones de la competencia mediática, tomadas como indicadores de las dimensiones del instrumento de evaluación que se presenta. (PérezEscoda,García-Ruiz y Aguaded, 2018, p.1)

Sin olvidar tampoco el peso del trabajo en equipo, como han demostrado estudios como los de López Vidales y González (2014), que implica "construir un proyecto colaborativo, aumentando su grado de implicación y responsabilidad en las tareas y trabajando en su capacidad de autocrítica para el desarrollo de un proyecto profesional real" (p.52). 
Por todo ello, el presente proyecto educativo recoge el 'guante didáctico' lanzado por estos autores, basándose también en los estudios acerca del papel del estudiante en el Aprendizaje Basado en Proyectos, ABP. Rodríguez, Luna y Vargas (2010), Pérez, Romero y Romeu (2014), o Bueno (2018) que insisten en la necesidad de cambiar el aprendizaje pasivo por la implicación del alumnado.

\subsection{Metodología}

La radio de la Universidad Europea Miguel de Cervantes es la más joven de las cinco existentes en Castilla y León. Frente a la veteranía de emisoras como la leonesa o salmantina, con dos décadas de trayectoria, esta emisora nace en la primavera de 2017. Pocos meses después, el coordinador propone a varios profesores trasladar las exposiciones orales de los trabajos grupales encomendado a su alumnado a la emisora. Esta propuesta pretendía sacar a los estudiantes del área de confort conformada por su espacio habitual lectivo (el aula) y los recursos expositivos tradicionales (proyector y ordenador), obligándoles a ser divulgativos y concisos en sus contenidos, además de tener que guionizar sus intervenciones y cuidar su voz. Todas las intervenciones se grabaron y colgaron en la web de la emisora (radio.uemc.es), vía podcast.

En el curso 2017-2018, tras varias reuniones con todos los departamentos académicos, dos docentes del área de Ciencias Sociales decidieron participar, modificando los ítems correspondientes en sus guías docentes. En su desarrollo curricular, explicaron a sus pupilos que el trabajo grupal de la materia se expondría en el estudio de radio. En torno a tres semanas antes de la citada exposición, cada docente se reunió con el responsable del proyecto para plantearle dudas de los estudiantes y de ellos mismos, de cara a asesorar al alumnado de la forma más eficaz posible.

Este mismo esquema se ha planteado en el curso 2018-2019. Uno de los docentes repitió y otro decidió plantear otro tipo de trabajo, por lo que descartó su adhesión. A cambio, otra profesora se unió. Las asignaturas que imparten tienen una eminente carga teórica (tabla 1). Cada grupo constó de entre tres y cinco miembros, en función del tamaño de la clase (que ha oscilado entre los 15 y los 25 alumnos), generándose 32 podcasts. 
Tabla 1

Disposición de las sesiones de exposiciones grupales en el estudio de radios

\begin{tabular}{|c|c|c|c|}
\hline Año académico & Asignatura / Grado & Temática & $\begin{array}{c}N^{\circ} \text { de podcasts } \\
\text { emitidos }\end{array}$ \\
\hline $2017-2018$ & $\begin{array}{c}\text { Recursos Humanos / } \\
\text { (ADE) }\end{array}$ & $\begin{array}{c}\text { Analizar y divulgar } \\
\text { informes en inglés sobre } \\
\text { retos de futuro del área } \\
\text { de Recursos Humanos }\end{array}$ & 4 \\
\hline $2017-2018$ & $\begin{array}{c}\text { Teoría de la } \\
\text { Comunicación / } \\
\text { Periodismo, Publicidad y } \\
\text { Com. Audiovisual }\end{array}$ & $\begin{array}{c}\text { Analizar tendencias de } \\
\text { medios de comunicación } \\
\text { de masas en su relación } \\
\text { con el impacto social de } \\
\text { algunos contenidos } \\
\text { periodísticos o } \\
\text { publicitarios }\end{array}$ & $\begin{array}{c}5 \text { (Publicidad) y } 7 \\
\text { (Periodismo y } \\
\text { Audiovisual) }\end{array}$ \\
\hline 2018-2019 & $\begin{array}{c}\text { Recursos Humanos / } \\
\text { (ADE) }\end{array}$ & $\begin{array}{l}\text { Explicar estudios } \\
\text { internacionales } \\
\text { simulando ser } \\
\text { empresarios o } \\
\text { consultores. }\end{array}$ & 6 \\
\hline 2018-2019 & $\begin{array}{l}\text { Historia Política y Social } \\
\text { Contemporánea / } \\
\text { Periodismo, Publicidad y } \\
\text { Com. Audiovisual }\end{array}$ & $\begin{array}{c}\text { Explicar la relevancia de } \\
\text { diversas figuras } \\
\text { femeninas, en ocasiones } \\
\text { poco desarrolladas en } \\
\text { los manuales de Historia }\end{array}$ & $\begin{array}{c}5 \text { (Publicidad) y } 5 \\
\text { (Periodismo y } \\
\text { Audiovisual) }\end{array}$ \\
\hline
\end{tabular}

Fuente: Elaboración propia.

Han participado en total 99 alumnos: 28 pertenecen al Grado en ADE (28,2\%), otros tantos a Publicidad, 25 a Comunicación Audiovisual y 18 a Periodismo. Son 49 mujeres y 50 hombres, con edades comprendidas entre los 18 y 21 años en un $87,9 \%$ de los casos, siendo el $12,1 \%$ restante alumnos de $22,23,25$ y 28 años.

Cada profesor explicó que entre el $20 \%$ y el $25 \%$ de la calificación del trabajo devendría de su exposición, a realizar en la emisora de radio, solo provistos con un guion de contenidos. Se les subrayó que los audios se colgarían en la web de la radio 
universitaria, para ser escuchados a posteriori por sus compañeros o cualquier otro tipo de público, por lo que debían exigirse ser divulgativos y expresarse con claridad y fluidez.

Una vez finalizada la experiencia, a cada estudiante se le pasó una encuesta anónima de diez preguntas, dirigidas en torno a su experiencia previa en radio, el interés y utilidad de la iniciativa, aspectos positivos y negativos, propuestas de mejora y una autoevaluación final. Es un análisis mixto basado en datos tanto cuantitativos como cualitativos, que continuó en los profesores, a quienes se les solicitó las calificaciones de la exposición grupal, además de entrevistarles de manera individual para correlacionar los datos obtenidos de las encuestas con su percepción.

El planteamiento diseñado ha querido cotejar las vertientes del proyecto, desde su utilidad hasta el impacto académico, pasando por la asunción de competencias comunicativas. Por la propia idiosincrasia de la institución educativa, los grupos son reducidos, siendo interesante, por un lado, la incorporación de nuevos miembros a la muestra; y, por el otro, la posibilidad de repetir la experiencia a los mismos alumnos en cursos superiores y en asignaturas de la misma índole que las analizadas.

\section{RESULTADOS}

Tras la grabación y emisión de los 32 podcasts, los 99 alumnos participantes trasladaron sus valoraciones a la encuesta diseñada. Menos de la mitad (un 44,4\%) sí habían realizado una experiencia similar en años previos (esencialmente en Bachillerato y en su primer año universitario, 38 de los 44 , es decir, un $86,3 \%$, y muy pocos durante su etapa de Secundaria). Un total de 29 estudiantes consideraron que esta experiencia les había resultado más difícil que una exposición oral en clase, 44 consideraron que tenía el mismo nivel de dificultad (la respuesta más común), y los 26 restantes entendieron que era más fácil.

Es ilustrativo conocer sus justificaciones. Por eso, tomando este trinomio de grado de dificultad como referencia, y añadiendo la variable de experiencia previa en la radio, y los cuatro Grados estudiados, se generan 24 combinaciones posibles reflejadas en la tabla 2, comprobándose antes que todas las combinaciones posibles de las variables habían escogido los tres grados de dificultad planteados. 
Tabla 2

Justificación del grado de dificultad según el grado estudiado y la experiencia previa en radio

\begin{tabular}{|c|c|c|c|}
\hline Grado cursado & $\begin{array}{c}\text { Experiencia previa } \\
\text { en radio }\end{array}$ & $\begin{array}{l}\text { Dificultad } \\
\text { seleccionada }\end{array}$ & $\begin{array}{c}\text { Justificación de la dificultad } \\
\text { seleccionada }\end{array}$ \\
\hline ADE & sí & FÁCIL & $\begin{array}{l}\text { "Al no preparar el PowerPoint, } \\
\text { fue más sencillo" }\end{array}$ \\
\hline ADE & sí & IGUAL & $\begin{array}{l}\text { "Solo cambia la forma de } \\
\text { transmitir el trabajo" }\end{array}$ \\
\hline ADE & sí & DIFÍCIL & $\begin{array}{l}\text { “Hay que preparar más a } \\
\text { conciencia de toda la } \\
\text { intervención” }\end{array}$ \\
\hline ADE & NO & FÁCIL & $\begin{array}{l}\text { "Era menos tiempo que en } \\
\text { clase y además en grupo" }\end{array}$ \\
\hline ADE & NO & IGUAL & “La preparación es la misma” \\
\hline ADE & NO & DIFÍCIL & “El micrófono imponía” \\
\hline PUBLICIDAD & sí & FÁCIL & $\begin{array}{l}\text { “Es más fácil porque las } \\
\text { presentaciones en clase son } \\
\text { aburridas" }\end{array}$ \\
\hline PUBLICIDAD & sí & IGUAL & "Nunca me pongo nervioso" \\
\hline PUBLICIDAD & sí & DIFÍCIL & “Mucha presión” \\
\hline PUBLICIDAD & NO & FÁCIL & $\begin{array}{l}\text { "No estar delante de tantas } \\
\text { personas físicamente" }\end{array}$ \\
\hline PUBLICIDAD & NO & IGUAL & $\begin{array}{l}\text { "Sólo se diferencia el modo } \\
\text { de presentar mi trabajo" }\end{array}$ \\
\hline PUBLICIDAD & NO & DIFÍCIL & $\begin{array}{l}\text { "Al ser en radio, tenía que ser } \\
\text { más profesional" }\end{array}$ \\
\hline PERIODISMO & sí & FÁCIL & "Me gusta la radio" \\
\hline PERIODISMO & sí & IGUAL & $\begin{array}{l}\text { "Podría ser más difícil porque } \\
\text { coincide con exámenes, pero } \\
\text { eso no lo hace más difícil” }\end{array}$ \\
\hline
\end{tabular}




\begin{tabular}{|c|c|c|c|}
\hline PERIODISMO & sí & DIFÍCIL & $\begin{array}{l}\text { "Nervios pueden traicionar y } \\
\text { resulta más difícil estructurar } \\
\text { discurso" }\end{array}$ \\
\hline PERIODISMO & NO & FÁCIL & "Es diferente y motiva" \\
\hline PERIODISMO & NO & IGUAL & $\begin{array}{l}\text { "Hay que esforzarse lo } \\
\text { mismo" }\end{array}$ \\
\hline PERIODISMO & NO & DIFÍCIL & $\begin{array}{l}\text { “Es más difícil porque al ser } \\
\text { radio me dirigía a un público } \\
\text { general que me puede } \\
\text { escuchar, no sólo a mis } \\
\text { compañeros” }\end{array}$ \\
\hline COM.AUDIOVISUAL & sí & FÁCIL & $\begin{array}{l}\text { “Es un ambiente profesional } \\
\text { el del estudio, que ayuda a } \\
\text { meterse en situación” }\end{array}$ \\
\hline COM.AUDIOVISUAL & sí & IGUAL & $\begin{array}{l}\text { "Estoy acostumbrado a } \\
\text { realizar exposiciones, así que } \\
\text { no me parecía diferente" }\end{array}$ \\
\hline COM.AUDIOVISUAL & sí & DIFÍCIL & $\begin{array}{l}\text { "Presión para evitar errores a } \\
\text { hablar" }\end{array}$ \\
\hline COM.AUDIOVISUAL & NO & FÁCIL & "No hace falta memorizar" \\
\hline COM.AUDIOVISUAL & NO & IGUAL & “Es otro formato, nada más” \\
\hline COM.AUDIOVISUAL & NO & DIFÍCIL & $\begin{array}{l}\text { "Tenía que prestar atención a } \\
\text { las palabras que decía” }\end{array}$ \\
\hline
\end{tabular}

Fuente: Elaboración propia.

Independientemente del Grado -aunque sin perder de vista que los vinculados a la Comunicación requieren per se de habilidades comunicativas, aunque éstas se desarrollan de forma progresiva durante los cuatro cursos- algunas respuestas, especialmente las de los pupilos decantados por la dicotomía 'fácil / difícil', son significativas; en tanto que atisban algunos de los parámetros establecidos en el planteamiento del proyecto.

Desde la alumna que entiende que es más complejo exponer un trabajo prestando más atención a las palabras, o quien se sentía presionado al saber que se le grababa 
su intervención o porque era consciente de que personas ajenas a sus compañeros podrían escuchar el podcast, pasando por el estudiante que se ha encontrado más motivado al encontrar en el estudio un ambiente profesional, o la persona que celebraba no tener que memorizar el contenido al estar guionizado.

Respuestas abiertas, en definitiva, que sintonizan con las premisas de nuevas fórmulas de aprendizaje y con la esencia de la radio.

\subsection{Significación y relevancia de la experiencia}

En una escala de 0 a 10, los 99 componentes de la muestra han calificado con un 8,31 de media el interés del proyecto, y con medio punto menos, exactamente un 7,831 , su utilidad. No se han apreciado diferencias significativas en la disección de estas puntuaciones en cuanto a Grados, salvo que los alumnos de Administración y Dirección de Empresas han otorgado notas más bajas (7,7 en interés y 6,82 en utilidad computando los resultados de ambos cursos) que los otros tres Grados, que están directamente vinculados al ámbito de la Comunicación (en todos ellos su grado de interés ha sido calificado con entre 8,5 y el 8,7 ; y la utilidad entre el 7,9 y el 8,5 ). Dicha vinculación se erige en el potencial factor de explicación de estas diferencias que, por otro lado, no son excesivas al hallarse en torno a un punto sobre diez).

Profundizando en la utilidad, se les pidió que escogieran qué aspecto les había resultado más provechoso, pudiendo marcar todas las opciones, alguna o ninguna: quitarse el miedo a hablar en público, exigirse concisión, capacidad de divulgar, tener a mano las intervenciones de los compañeros para ayudar en el estudio y romper la dinámica de las clases.

La opción preferente, destacada sobre el resto, ha sido la última. Un total de 85 de los 99 encuestados opinaron que la intervención en el estudio suponía una dinámica diferente y les obligaba a salir del aula, con porcentajes muy similares en los cuatro Grados analizados. Le siguen, a bastante distancia porcentual, la autoexigencia a explicar los contenidos de forma divulgativa (44\%), la concisión $(34,3 \%)$ y quitarse el miedo a hablar en público (33,3\%). Menos del $10 \%$ entendió que la iniciativa le servía para escuchar a sus compañeros en otro momento para ayudar a refrendar los contenidos teóricos.

En los cuatro Grados el orden clasificatorio de las cinco opciones ha sido similar, con dos excepciones: en Publicidad, casi el 50\% anotó la opción de vencer la barrera de hablar en público (en el resto de Grados este porcentaje está entre el 25 y el $33 \%$ ), convirtiéndose en la tercera opción de las cinco; en tanto que, en Periodismo, la segunda elección mayoritaria no ha sido la referente a la divulgación (que, en este 
caso, ocupa el cuarto lugar, con un $27,7 \%$ ), sino la búsqueda de concreción a la hora de transmitir el contenido de forma oral, con un 55,5\%, el doble respecto al ítem de divulgación.

De estos datos se desprende que los alumnos han valorado unánimemente y de forma positiva salir de su área de confort -una inquietud de los docentes en las reuniones previas-, en tanto que, en cada Grado se ha extraído de forma particular otro aprovechamiento: aprender a ser divulgativos (en todos los casos salvo en Periodismo, dado que esta competencia se trabaja desde el inicio del Grado y los estudiantes han hallado menos dificultades en este reto) y perder el miedo a hablar en público. Puede resultar sintomático que esta opción haya sido la tercera en su orden de preferencia, dado que en las exposiciones no tenían que enfrentarse a un público (el resto de sus compañeros), sino que solo estaban presentes los componentes de su grupo. Sin embargo, al interrelacionar esta idea con las respuestas brindadas en la pregunta abierta sobre el grado de dificultad, se constata que, a pesar de no tener oyentes en directo, la emisora les generó un extra de presión al no contar con apoyo visual y planear sobre ellos la tensión al ser conscientes de que estaban siendo grabados.

Esta idea encuentra solución de continuidad al explorar los puntos débiles detectados por los alumnos. Antes de pedirles una autocalificación de seis aspectos vinculados a la comunicación, se les solicitó en la encuesta que escogiesen cuál había sido el aspecto más complejo al que se habían enfrentado en esta exposición oral.

La mayoría refutó el apriorismo con el que entraron en la emisora. En las pruebas de sonido, muchos manifestaron que echaban en falta su presentación en PowerPoint. Pero tras cumplimentar la encuesta, ésta fue la elección minoritaria (tabla 3). 
Tabla 3

Principal dificultad detectada en la grabación de los podcasts según grado

\begin{tabular}{|l|l|l|l|l|}
\hline \multicolumn{1}{|c|}{$\begin{array}{c}\text { Ítem / Grado } \\
\text { (n' } \text { alumnos) }\end{array}$} & \multicolumn{1}{|c|}{ ADE (28) } & $\begin{array}{c}\text { Periodismo } \\
\mathbf{( 1 8 )}\end{array}$ & Publicidad (28) & Audiovisual (25) \\
\hline $\begin{array}{l}\text { Prestar atención a mi } \\
\text { dicción (pronunciación } \\
\text { de las palabras) }\end{array}$ & $10(35,7 \%)$ & $5(27,7 \%)$ & $10(35,7 \%)$ & $8(32 \%)$ \\
\hline $\begin{array}{l}\text { Miedo a equivocarme al } \\
\text { ser contenido grabado }\end{array}$ & $10(35,7 \%)$ & $2(11,1 \%)$ & $6(21,4 \%)$ & $6(24 \%)$ \\
\hline $\begin{array}{l}\text { Tener que emplear un } \\
\text { lenguaje diferente, } \\
\text { divulgativo }\end{array}$ & $3(10,7 \%)$ & $4(22,2 \%)$ & $9(32,1 \%)$ & $9(36 \%)$ \\
\hline $\begin{array}{l}\text { Estar en un entorno } \\
\text { diferente }\end{array}$ & $4(14,2 \%)$ & $3(16,6 \%)$ & $3(10,7 \%)$ & $2(8 \%)$ \\
\hline & $1(3,6 \%)$ & $4(22,2 \%)$ & 0 & 0 \\
\hline Carecer de apoyo visual
\end{tabular}

Fuente: Elaboración propia.

En términos generales, el aspecto de la dicción ha sido el que más impactado en los cuatro Grados, con un tercio de las respuestas (33 de las 99), y ha sido también la primera en el ranking individual de cada Grado. Le siguen el aspecto de la divulgación, con 25 , seguido muy de cerca por el miedo que les generaba el saberse grabados, con 24, y lejos quedan los aspectos de la novedad del entorno, con 12, y el apoyo visual, con 5 .

En la misma línea, los alumnos ajenos al área de Comunicación (ADE) han sido, con diferencia, los que más han remarcado su tensión al saber que su intervención sería registrada; en el polo opuesto, los periodistas han revelado que se sintieron cómodos, algo natural teniendo en cuenta que en su futuro laboral éste puede ser uno de sus escenarios de trabajo.

En correlación con los puntos fuertes antes explicados, este análisis ha revelado que uno de cada tres estudiantes del total de la muestra manifestó que la actividad les había servido para despojarse del miedo a hablar en público. Es plausible denotar que 
ambas vertientes (logro conseguido y dificultad detectada) van en consonancia, como también lo es otra de las fortalezas asimiladas, la divulgación; aunque, en este caso, solo en Audiovisual y Publicidad reconocieron que les costó bastante ejecutarlo (más de un $30 \%$ de coincidencias, dato superior al de los otros dos Grados).

Han sido los estudiantes de Periodismo los que más han echado en falta un soporte visual (4 de las 5 respuestas), creando un particular constructo: no se encontraron incómodos en la emisora, donde quizá trabajen en el futuro, se vieron divulgativos, pero se vieron despojados del factor imagen para llegar a su público.

Pero para acotar de forma nítida estos datos, se acude ahora a una valoración más profunda de las habilidades desarrolladas, introduciendo además el elemento docente como factor para generar la contrastación.

\subsection{Autoevaluación y heteroevaluación}

Seis aspectos compusieron la doble evaluación (la interna de cada alumno, y la externa, procedente de su docente), ponderada de 0 a 10 puntos en cada uno: dominio de los conceptos de la asignatura, capacidad de explicar dichos conceptos de forma adecuada, ser dinámico, nivel de pronunciación, dominio de nervios y estructura lógica del discurso. Estos aspectos emanan consecuentemente de los puntos fuertes y débiles analizados y de la esencia de los mecanismos de transmisión de contenidos orales propios del medio radio. Cada alumno, por tanto, se evaluó de forma autónoma (e independiente de su calificación en la prueba, dado que la conoció semanas más tarde), y cada docente puntuó a toda la clase, de forma general, en este intervalo numérico de 0-10; para, así, poder establecer correlaciones objetivas (tabla 4).

Tabla 4

Autoevaluaciones generales y por grado de los aspectos desarrollados

\begin{tabular}{|l|l|l|l|l|l|}
\hline \multicolumn{1}{|c|}{ Grado } & \multicolumn{1}{|c|}{ ADE } & Periodismo & Publicidad & Audiovisual & Medias \\
\hline \multicolumn{1}{|c|}{ Ítem } & Alum-Prof & Alum-Prof & Alum-Prof & Alum-Prof & Alum-Prof \\
\hline $\begin{array}{l}\text { Dominio de los } \\
\text { conceptos de la } \\
\text { asignatura }\end{array}$ & $69-6$ & $6,89-8$ & $7,4-8$ & $7,36-8$ & $7,162-7,5$ \\
\hline Capacidad de & $7,35-6$ & $7,02-6,5$ & $8,07-7,5$ & $7,53-6,5$ & $7,535-6,63$ \\
\hline
\end{tabular}




\begin{tabular}{|l|l|l|l|l|l|}
\hline explicación & & & & & \\
\hline Dinamismo & $6,85-5$ & $7-8$ & $7,64-8,5$ & $7,28-8$ & $7,212-7,37$ \\
\hline Dicción & $7,36-7$ & $7,39-7,5$ & $7,57-7,5$ & $7,16-7,5$ & $7,374-7,37$ \\
\hline Dominio de nervios & $7,5-7$ & $7,22-7$ & $7,36-7$ & $7,68-7$ & $7,455-7$ \\
\hline Estructura del discurso & $7,31-6$ & $7,5-6,5$ & $8,29-7$ & $7,64-6,5$ & $7,707-6,5$ \\
\hline
\end{tabular}

Fuente: Elaboración propia.

No existen grandes contrastes numéricos. Esta cercanía entre autoevaluación y heteroevaluación implica los alumnos fueron objetivos, incluso críticos, con su visión acerca de la experiencia radiofónica universitaria. Premisa a tener en cuenta dado que, por lo general, un estudiante suele verse mejor en las pruebas que ejecuta respecto a lo que considera su profesor. Bien es cierto, empero, que en las 30 comparativas (seis ítems y cinco áreas), en dos de cada tres (20), el alumnado se ha valorado mejor respecto al docente.

Las mayores diferencias (de más de un punto) llegan en el apartado 'estructura del discurso' -en los cuatro Grados, con percepciones siempre superiores en el caso del alumnado-, seguida de la capacidad de ser explicativos; pero, en el otro extremo de la balanza, son varios los casos en los que la distancia es inferior a medio punto (muy significativo el caso de la dicción, en el que la resta arroja muy pocas décimas de diferencia).

El segundo punto a subrayar es que, revisando los datos a nivel global, los 99 alumnos se calificaron con una nota media superior al 7 e inferior al 8 en los seis aspectos ponderados. En otras palabras, de forma general, los alumnos se conceden un notable, difiriendo sólo de la visión de sus docentes en dos aspectos (explicación y estructura, en los que se les valora con 6,5 puntos).

Los estudiantes de ADE se ubicaron como un grupo que supo explicarse bien, dominó los nervios y ejecutó un discurso coherente, pero a cambio, observó fisuras en su capacidad de ser dinámicos y su conceptualización de la materia, aspecto este último en el que también se valoró poco el grupo de Periodismo, al que se puede etiquetar como homogéneo en su visión (apenas medio punto de diferencia en los otros cinco ítems). Los publicistas han sido el grupo con una mejor valoración de sí mismos, con las notas más altas; y, curiosamente, también han recibido la mejor tasación de su profesorado (ninguna nota ha sido inferior al 7). 
Además de su ponderación global de los seis aspectos, los docentes también evaluaron sus intervenciones mediante rúbrica. Se instó a todos ellos a que los elementos evaluados fuesen los seis que conforman este análisis, aunque se dejó a criterio de cada profesor el peso relativo de cada ítem en la nota final, para no interferir en su labor.

Apenas se registró un suspenso en los 32 grupos evaluados, y las notas medias oscilaron entre los 6,5 y los 7,5 puntos, con alguna excepción. En el Grado en ADE, en el curso 2017-2018 la media fue de un 6,75; al año siguiente 7,88 puntos.

En el caso de Periodismo, Comunicación Audiovisual y Publicidad, cabe recordar que las calificaciones de cada curso académico proceden de dos docentes diferentes. Además, las notas de Periodismo y Audiovisual son las mismas, dado que ambos Grados se unifican en la misma aula durante la primera parte de su etapa universitaria.

En el curso 2017-2018 los grupos de Periodismo y Audiovisual obtuvieron una calificación de 6,78, y sus compañeros de Publicidad, un 9. El planteamiento del trabajo era idéntico en ambas clases (por tratarse de la misma asignatura), aunque la temática a desarrollar fue diferente (se adaptó a los contenidos temáticos curriculares de cada carrera). La nota media global, uniendo los doce grupos de los tres Grados fue de 7,71 puntos.

En el curso 2018-19 comparecieron cinco grupos de la clase de Periodismo y Audiovisual, con notas entre 6 y 7 puntos (promedio de 6,65); en tanto que se formaron seis grupos de trabajo en Publicidad, con calificaciones dispares entre la máxima (8) y la mínima (5), dando como media un 6,5. La media global de este curso académico es de 6,57 .

\subsection{Cotejo de las valoraciones}

El último puntal de esta estructura procede de las entrevistas en profundidad generadas a los docentes implicados, y en las respuestas abiertas del alumnado acerca de si consideraba interesante repetir, por qué y qué mejorarían. Para agilizar el discurso de este punto, se catalogarán las respuestas de cada docente con un código simple compuesto de la letra $\mathrm{D}$ y un número del 1 al 3 ; en el caso de los alumnos, con la A mayúscula y su número de codificación (entre el 1 y el 99).

A los alumnos, por lo general, les resultó útil y eficaz esta propuesta. Abundaron en sus respuestas a la mencionada pregunta de carácter abierto que les gustaría repetir porque rompía la monotonía (10 coincidencias) y era ameno y original (7 coincidencias). Dos de los 99 encuestados no respondieron a la cuestión de si consideraban interesante repetir, y otra persona respondió que no, sin indicar motivos. 
Los docentes también consideraron que el proyecto fue "novedoso" (D1) porque "motivó al alumnado" (D3), dado que "ya que han hecho muchas exposiciones en clase, y de esta forma, pueden presentárselo a sus familias en casa desde la web de la universidad y mostrar lo que hacen" (D2). Aspecto corroborado por los estudiantes: "ha servido para que llegue a la población lo que estudiamos" (A32), "intentando adaptar lo que decimos a un público que no conoce nuestra materia" (A38).

En la vertiente académica, un profesor postuló que la utilidad residía en "no relajarme en el método [...] para también añadir a mi materia los temas de redacción y exposición" (D2), y algunos estudiantes reseñaron que "ayuda a interesarte por la asignatura, que es teórica" (A88), "adaptar materias menos atractivas a formatos como éste mejora el interés" (A73), "todo el mundo debería probar esto, independientemente del Grado que estudie" (A97). Otros buscaron la vertiente del horizonte laboral al considerar el proyecto "una adaptación a una situación nueva y diferente" (A51), o "un simulacro de cara a futuro" (A40), e incluso "se cogen ideas de otro entorno profesional, eso es bueno para futuro" (A28).

Un entorno, por otra parte, desconocido para una importante porción de la muestra, por lo que los docentes coincidieron en la idoneidad de celebrar un taller previo para resolver preguntas, "dudas por su inexperiencia en este medio" (D1), aunque también concedieron en expandir la planificación previa mediante "pequeñas instrucciones previas de qué es hablar en la radio, por su inexperiencia general" (D3). Una inexperiencia, no obstante, que consideraron que fue positiva, ya que "estuvieron muy involucrados intentado hacer algo más que una exposición, intentaron transformar el formato del examen en una entrevista o tertulia, aunque esto les conllevara más trabajo", "vieron que se auto responsabilizaban de su trabajo y resultado" (D3), "la inmersión de los alumnos en un escenario profesional, la experiencia de juego y la tutela de la presentación por parte de un profesional del medio" (D2).

A pesar de las positivas valoraciones tanto numéricas como en las opiniones recabadas, los profesores consideran que algunos estudiantes se tomaron esto "demasiado lúdico" (D1), necesitando "preparar mejor, en algunos casos, el contenido y la estructura de su discurso" (D1), "sintetizando más y siendo creativos" (D2), "en algunos puntos sólo han trabajado en grupo (cada uno ha hecho lo suyo y lo han juntado delante del micrófono) [...] el formato radiofónico lo propiciaba pero no han aprovechado la oportunidad" (D3). Por ello, piensan que es bueno fomentar una mayor aproximación previa al medio radio (en esencia, cómo hablar y cómo escribir) para mejorar el aprovechamiento de la práctica. 


\section{DISCUSIÓN Y CONCLUSIONES}

Buscar la innovación educativa debe ser una constante en la tarea docente para implicar a los alumnos. Esta experiencia ha pretendido aunar bajo un mismo paraguas los requisitos del Plan Bolonia, expandir las potencialidades de una radio universitaria, y sumergir a los alumnos en la adquisición de competencias vinculadas a la comunicación. Los docentes deben insistir en que, independientemente del grado que se curse, transmitir contenidos de forma oral es un ítem fundamental (por ejemplo, para una entrevista de trabajo, dar una charla a un público general o especializado, vender una idea de negocio...). Esta experiencia ha tratado de aprovechar la excusa de asignaturas más teóricas para romper con la dinámica habitual e instar a los alumnos a salir del área de confort.

Los resultados han mostrado que los alumnos han incrementado su motivación por las materias que se han adherido al proyecto. Materias que han implementado una nueva fórmula de desarrollo docente, acorde con las exigencias del Espacio Europeo de Educación Superior, convirtiendo la sesión académica en una simulación de entorno real.

Consecuentemente, se han responsabilizado más, al saber que otras personas podrían escucharles. Han sido críticos con su labor, como constatan las calificaciones de autoevaluación, convergentes con las notas de sus profesores, y las valoraciones aportadas por las entrevistas realizadas, confirmando así las dos hipótesis de partida.

El objetivo general de la investigación perseguía dirimir el grado de consecución de competencias vinculadas a la comunicación oral. Si bien es cierto que quienes más trabajarán esta faceta serán los periodistas, no cabe duda de que publicistas, comunicadores audiovisuales y gestores empresariales deben tener en cuenta esta premisa.

El estudio se ha realizado a estudiantes de primer y segundo curso, sin nociones en esta materia. Consecuentes a la responsabilidad que auto-asumieron, se volcaron en ser originales, directos, concisos, pero a la vez divulgativos. Armas necesarias en la comunicación oral en general, que se manifiestan de forma más explícita aún en el medio radio tradicional, carente del factor imagen y la comunicación no verbal.

De igual modo, se desarrollan otras capacidades, como resolución de problemas, el trabajo en equipo, la planificación, la toma de decisiones o el saber compartir, si bien es cierto que, tal y como se ha plasmado, algún docente se percató de que algunos grupos funcionaron mal, de ahí las calificaciones más bajas. 
Se ha podido constatar el impacto que supone el medio radiofónico en los estudiantes, sean o no del área de Comunicación, tengan o no experiencia en estos formatos, no sólo en lo referente al cambio de hábitat y la ruptura con las rutinas habituales de asignaturas etiquetadas como teóricas, sino también en el desarrollo de su potencial comunicativo.

Se asumen algunas propuestas de mejora que han emanado de las respuestas. Generar una pequeña formación previa a los estudiantes pueden ayudarles a vencer el miedo escénico ante el escenario de exposición pública y a dotarles de herramientas básicas para perfeccionar su discurso. De cara a futuro, también se puede plantear que los podcasts no 'naveguen' por la red de redes de forma independiente, sino que conformen una narrativa con discurso propio; en otras palabras, conformar con todos los docentes una especie de leit motiv general y unitario, a modo de programa temático radiofónico, sobre el que cada disciplina aporte sus conocimientos en varias secciones.

\section{Referencias}

Chenovart-González, J. y Castelló-Belda, R. (2018). Formación radiofónica universitaria en el modelo transmedia: el caso de Ràdio Universitat (2011-2015). Revista Mediterránea de Comunicación/Mediterranean Journal of Communication, 9(1), 97-116. DOI: https://www.doi.org/10.14198/MEDCOM2018.9.1.6

Barranquero, A. (2007). "Concepto, instrumentos y desafíos de la edu-comunicación para el cambio social". Comunicar. Revisa científica iberoamericana de comunicación y educación, 29, 115-120.

De Semir, V. (2003). Medios de comunicación y cultura científica. Quark: Ciencia, medicina, comunicación y cultura, (28-29), 22-34. Recuperado de: https://goo.gl/sCHPH6

Díaz Monsalvo, M.Á. (2018). El perfil profesional en radio hoy. Aplicación de las competencias de los Grados de Periodismo y Comunicación en las emisoras universitarias de Castilla y León. (Trabajo Fin de Máster). Universidad de Valladolid, España. Disponible en web: http://uvadoc.uva.es/handle/10324/33348

González, V. \& Contreras-Pulido, P. (2014). Empoderar a la ciudadanía mediática desde la educomunicación. Empowering Media Citizenship through Educommunication. Comunicar, 42, (XXI), 2014, p. 129-136. DOI: http://dx.doi.org/10.3916/C42-2014-12

Kaplún, M. (1998). Una pedagogía para la comunicación. Madrid: Ediciones de la Torre. 
López Vidales, N., y Gómez Rubio, L. (2014). La democratización del proceso comunicativo en radio: los jóvenes prosumidores. Vivat Academia, 17(126), 31-53. Recuperado de: http://www.vivatacademia.net/index.php/vivat/article/download/552/79

López Vidales, N., Gómez Rubio, L., y Hernando Lera, M. (2015). Las radios universitarias, ¿escaparate de la radio del futuro? Los jóvenes como creadores de contenidos radiofónicos. En J. Herrero, y C. Mateos (coords). La pantalla insomne. Actas del VII Congreso Internacional Latina de Comunicación Social, 19-44. Universidad de La Laguna, Tenerife.DOI: 10.4185/cac90

López Vidales, N. y González, P. (2014). Audioblogs y Tvblogs, herramientas para el aprendizaje colaborativo en Periodismo. Comunicar, 21(42), 45-53.

Martín-Pena, D. Segura-Anaya, M., y Martín-Lazo, C. (2015). Profile of Contributors of University Radio in Spain: The Case of Students in the Area of Information Science. Studies in Media and Communication, 3(2), 164-171. DOI: https://doi.org/10.11114/smc.v3i2.1228

Martín-Pena, D. (2013). Radios universitarias en España: plataformas de comunicación interactiva y redes de colaboración (tesis doctoral). Universidad de Huelva, España. DOI: https://doi.org/https://doi.org/10.1017/CBO9781107415324.004

Morales Bueno, P. (2018). Aprendizaje basado en problemas (ABP) y habilidades de pensamiento crítico ¿una relación vinculante? Revista Electrónica Interuniversitaria de Formación del Profesorado, 21(2), 91-108. DOI: http://dx.doi.org/10.6018/reifop.21.2.323371

Morsy, Z. (Coord.) (1984). La educación en materia de comunicación. París, Unesco.

Ortiz Sobrino, M., Marta-Lazo, C. y Martín-Pena, D. (2016). La formación de competencias profesionales en los estudiantes de Comunicación Social de las emisoras universitarias en España y Portugal: situación y resultados asimétricos. Signo y Pensamiento, 35(68), 35-50. DOI: http:// dx.doi.org/10.11144/Javeriana.syp35-68.fcpe

Parejo-Cuéllar, M. M., Vivas-Moreno, A. y Martín-Pena, D. (2018). La divulgación científica en las emisoras universitarias. El caso de la fonoteca del proyecto Semillas de Ciencia. Revista Mediterránea de Comunicación/Mediterranean Journal of Communication, 9(1), 35-47. DOI: https://www.doi.org/10.14198/MEDCOM2018.9.1.2

Pérez-Escoda, A., García-Ruiz, R., Aguaded-Gómez, I. (2018). La competencia mediática en el profesorado universitario. Validación de un instrumento de evaluación. @tic revista d'innovació educativa, 21, 1-9. DOI: https://doi.org/10.7203/attic.21.12550

Pérez Mateo, M., Romero, M., \& Romeu-Fontanillas, T. (2014). La construcción colaborativa de proyectos como metodología para adquirir competencias digitales. Comunicar: Revista Científica Iberoamericana de Comunicación y Educación, 21(42), 15-23. DOI: https://doi.org/10.3916/C42-2014-01 
Pérez Tornero, J. M. (2008). Comunicación y Educación. Cuestiones clave. Mentor Media Literacy.

Pinto, R., Martín-Pena, D. y Vivas-Moreno, A. (2016): La radio universitaria como formadora de profesionales. Análisis de la relación entre los títulos de grado y la radio universitaria española. Revista General de Información y Documentación, 26(1), 221-248

Ponce López, $M^{a}$ C. (2019). Iniciación a los fundamentos teóricos para la educomunicación en la radio universitaria. Miguel Hernández Communication Journal, 10(1), 183-197. DOI: http://dx.doi.org/10.21134/mhcj.v10i0.269

Rodríguez-Sandoval, E., Luna-Cortés, J., \& Mauricio Vargas-Solano, E. (2010). Evaluación de la estrategia del aprendizaje basado en proyectos. Educación y Educadores, 13(1), 1325. DOI: https://doi.org/ISSN 0123-1294

Romero-Valderrama, A.C., Forero-Romero, A., y Rodríguez-Hernández, A. (2018). Análisis comparación del aprendizaje basado en proyectos de forma tradicional y con mediación de las TIC. Espacios, 39(52), 28-42.

Ruipérez-Almajano, $M^{a}$ N., Gutiérrez-Palmero, $M^{a}$ J., Olarte-Martínez, M., y Azofra-Agustín, E. (2011). Experiencia docente en la organización de una actividad práctica. Radio universidad como estrategia de aprendizaje y motivación. En. Ma.P. Pérez Bermúdez y A.Guillén-Riquelme (cords.). VIII Foro sobre Evaluación de la Calidad de la Investigación y de la Educación Superior: Libro de Capitulos. Granada: Asociación Española de Psicología Conductual (AEPC), 2011, 844-848

Sánchez-Carrero, J. \& Contreras-Pulido, P. (2012). De cara al prosumidor: producción y consumo empoderando a la ciudadanía 3.0. Icono 14, (10), 62-84. DOI: 10.7195/ri14.v10i3.210

Vargas-Guerrero, B., et al. (2018). Lectura y escritura a través de la investigación como estrategia pedagógica apoyada en las TIC. Cultura. Educación y Sociedad 9(3), 645654.

Vázquez Guerrero, M. (2012). La Radio universitaria en México y España. Estudio de la participación y formación de los jóvenes (tesis doctoral). Universidad Pompeu Fabra, Barcelona. Recuperado de http://www.tdx.cat/handle/10803/84113

Zamarra López, M. (2018). Radios universitarias: Plurales, abiertas y participativas. Revista Mediterránea de Comunicación/Mediterranean Journal of Communication, 9(1), 117135. DOI: https://www.doi.org/10.14198/MEDCOM2018.9.1.7 